\title{
NEAREST SOUTHEAST SUBMATRIX THAT MAKES MULTIPLE AN EIGENVALUE OF THE NORMAL NORTHWEST SUBMATRIX
}

\author{
JuAn-Miguel GRACIA AND FRAnCisco E. VELASCO
}

Abstract. Let $A, B, C, D$ be four complex matrices, where $D \in \mathbb{C}^{m \times m}$ and $A \in \mathbb{C}^{n \times n}$ is a normal matrix. Let $z_{0}$ be an fixed eigenvalue of $A$. We find the distance (with respect to the 2 -norm) from $D$ to the set of matrices $X \in \mathbb{C}^{m \times m}$ such that $z_{0}$ is a multiple eigenvalue of the matrix

$$
\left(\begin{array}{ll}
A & B \\
C & X
\end{array}\right) .
$$

We also give an expression for one of the closest matrices.

Mathematics subject classification (2010): 15A18, 15A60, 15A09.

Keywords and phrases: Singular values, analytic matrix functions, asymptotic behavior, Malyshev, Moore-Penrose inverse.

\section{REFERENCES}

[1] M. A. Beitia, I. DE Hoyos, I. Zaballa, The change of similarity invariants under row perturbations, Linear Algebra Appl. 429 (2008), 1302-1333.

[2] D. S. Bernstein, Matrix Mathematics, Princeton University Press, 2005.

[3] S. L. Campbell and C. D. Meyer, Generalized Inverses of Linear Transformations, Pitman, London, 1979 .

[4] G. CRAVO, Matrix completion problems, Linear Algebra Appl. 430 (2009), 2511-2540.

[5] K. DU, Y. WEI, Structured pseudospectra and structured sensitivity of eigenvalues, J. Comput. Appl. Math. 197 (2006), 502-519.

[6] J. M. GonzÁlez de DuranA, J. M. GraCiA, Geometric multiplicity margin for a submatrix, Linear Algebra Appl. 349 (2002), 77-104.

[7] J. M. GRACIA, Nearest matrix with two prescribed eigenvalues, Linear Algebra Appl. 401 (2005), 277-294.

[8] J. M. GRACIA, F. E. VELASCO, Nearest southeast submatrix that makes multiple a prescribed eigenvalue. Part 1, Linear Algebra Appl. 430 (2009), 1196-1215.

[9] D. HinRICHSEn, B. KELB, Spectral value sets: a graphical tool for robustness analysis, Systems Control Lett. 21 (1993), 127-136.

[10] D. Hinrichsen, A. J. Pritchard, Mathematical systems theory I, Springer, 2000.

[11] T. HU, L. QIU, On structured perturbation of Hermitian matrices, Linear Algebra Appl. 275-276 (1998), 287-314.

[12] A. N. MALYSHEV, A formula for the 2-norm distance from a matrix to the set of matrices with multiple eigenvalues, Numer. Math. 83 (3) (1999) 443-454.

[13] G. W. Stewart, J. G. Sun, Matrix perturbation theory, Academic Press, 1990.

[14] M. WEI, Perturbation theory for the Eckart-Young-Mirsky theorem and the constrained total least squares problem, Linear Algebra Appl. 280 (1998), 267-287. 